\title{
An eccentric wave in the circumstellar disc of the Be/X-ray binary X Persei
}

\author{
R. K. Zamanov, ${ }^{1 \star}$ K. A. Stoyanov ${ }^{1}$, U. Wolter ${ }^{2}$, D. Marchev ${ }^{3}$, N. A. Tomov ${ }^{1}$, \\ M. F. Bode ${ }^{4,5}$, Y. M. Nikolov ${ }^{1}$, V. Marchev ${ }^{1}$, L. Iliev ${ }^{1}$, I. K. Stateva ${ }^{1}$ \\ 1 Institute of Astronomy and National Astronomical Observatory, Bulgarian Academy of Sciences, 72 Tsarigradsko Shose, \\ 1784 Sofia, Bulgaria \\ 2 Hamburger Sternwarte, Universität Hamburg, Gojenbergsweg 112, 21029 Hamburg, Germany \\ ${ }^{3}$ Department of Physics and Astronomy, Shumen University, 115 Universitetska Str., 9700 Shumen, Bulgaria \\ 4 Astrophysics Research Institute, Liverpool John Moores University, IC2, 149 Brownlow Hill, Liverpool, L3 5RF, UK \\ 5 Office of the Vice Chancellor, Botswana International University of Science and Technology, Private Bag 16, Palapye, Botswana
}

Accepted 2020 September 30. Received 2020 September 18; in original form 2020 March 16

\begin{abstract}
We present spectroscopic observations of the Be/X-ray binary X Per obtained during the period December 2017 January 2020 (MJD 58095 - MJD 58865). In December 2017 the $H \alpha, H \beta$, and HeI 6678 emission lines were symmetric with violet-to-red peak ratio $V / R \approx 1$. During the first part of the period (December 2017 - August 2018) the V/Rratio decreased to 0.5 and the asymmetry developed simultaneously in all three lines. In September 2018, a third component with velocity $\approx 250 \mathrm{~km} \mathrm{~s}^{-1}$ appeared on the red side of the HeI line profile. Later this component emerged in $H \beta$, accompanied by the appearance of a red shoulder in $H \alpha$. Assuming that it is due to an eccentric wave in the circumstellar disc, we find that the eccentric wave appeared first in the innermost part of the disc, it spreads out with outflowing velocity $v_{\text {wave }} \approx 1.1 \pm 0.2 \mathrm{~km} \mathrm{~s}^{-1}$, and the eccentricity of the eccentric wave is $e_{\text {wave }} \approx 0.29 \pm 0.07$. A detailed understanding of the origin of such eccentricities would have applications to a wide range of systems from planetary rings to AGNs.
\end{abstract}

Key words: Stars: emission-line, Be - stars: winds, outflows - circumstellar matter - X-rays: binaries - stars: individual: X Per

\section{INTRODUCTION}

The relatively bright variable star X Persei (HD 24534) is the optical counterpart of the X-ray source $4 \mathrm{U} 0352+309$ (Braes \& Miley 1972) and is classified in the Be/X-ray subclass of massive X-ray binary stars (e.g. Negueruela 2007; Reig 2011). It consists of an early type Be star and a slowly spinning neutron star. The X-ray data revealed a neutron star with spin period $\approx 836 \mathrm{~s}$, which exhibits quasi-periodic X-ray flares with a period of $\sim 7$ years (Nakajima et al. 2019). The pulse period shows episodes of spin-up and spindown (Acuner et al. 2014) which indicates that the neutron star is close to a torque equilibrium and the long pulse period suggests a strong magnetic field (Yatabe et al. 2018). Delgado-Martí et al. (2001) determined the orbital period $\sim 250 \mathrm{~d}$, orbital eccentricity $e=0.11$, semi-major axis $a=$ 2.2 a.u and orbital inclination $i=26^{0}-33^{0}$.

During the last century, the visual brightness of $\mathrm{X}$ Per has varied in the range $\mathrm{V}=6.8$ to $6.2 \mathrm{mag}$. Spectrograms

^ E-mail: rkz@astro.bas.bg (RKZ), kstoyanov@astro.bas.bg (KAS)

(C) 2020? The Authors of X Per from 1913 to 2020 show X Per to have bright hydrogen lines that are variable in structure, velocity, and intensity (e.g. Cowley et al. 1972; Telting et al. 1998). The primary is a rapidly rotating main sequence Be star which forms an outwardly diffusing gaseous disc. It has projected rotational velocity $\approx 215 \mathrm{~km} \mathrm{~s}^{-1}$ (Lyubimkov et al. 1997), and is classified as O9.5 (Fabregat et al. 1992), B0Ve (Roche et al. 1997), B1Ve (Zamanov et al. 2019).

In recent decades, X Per exhibited two disc-loss episodes (around 1977 and around 1989) when the emission lines and the circumstellar disc were missing (Roche et al. 1997; Clark et al. 2001). The long-term variability of the emission lines and optical brightness indicate variable mass ejections from the donor star into the circumstellar disc ( $\mathrm{Li}$ et al. 2014).

We present optical spectroscopic observations obtained in the last two years and discuss asymmetries in the circumstellar disc and the appearance of eccentric wave. 

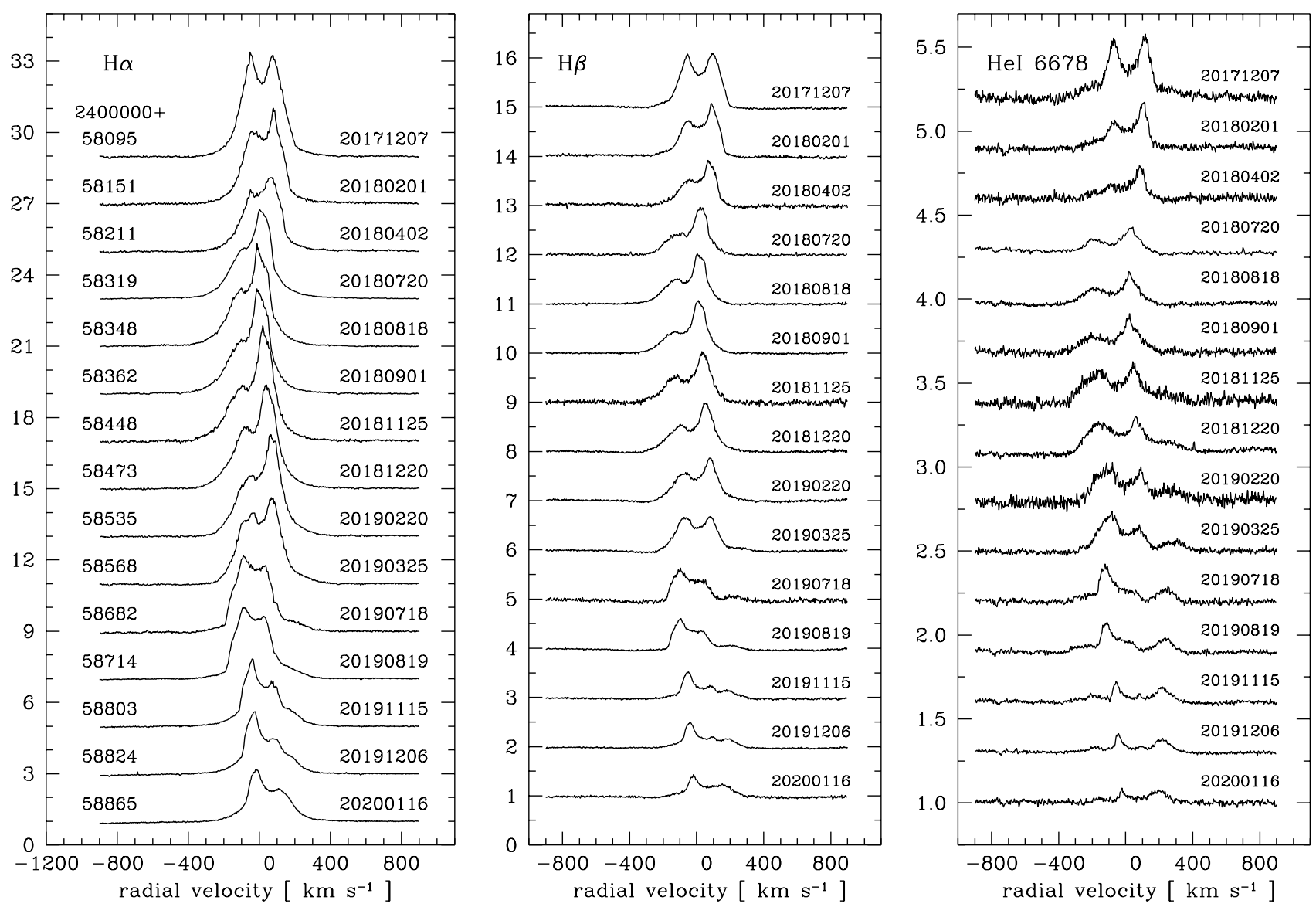

Figure 1. Variability of $H \alpha, \mathrm{H} \beta$ and HeI 6678 emission line profiles of X Per. The date of observations is in the format YYYYMMDD.

\section{OBSERVATIONS}

We have 101 optical spectra of X Per on 57 nights secured with the ESpeRo Echelle spectrograph (Bonev et al. 2017) on the $2.0 \mathrm{~m}$ RCC telescope of the Rozhen National Astronomical Observatory, Bulgaria and with the HEROS spectrograph (Schmitt et al. 2014) on the $1.2 \mathrm{~m}$ TIGRE telescope in the astronomical observatory La Luz in Mexico. The variability of $H \alpha, \mathrm{H} \beta$ and HeI 6678 emission lines of X Per is presented in Fig. 1. The spectra are normalized to the local continuum and a constant is added to each spectrum. In this figure are plotted only 15 out of 101 spectra obtained.

On the spectra we measure the following parameters: equivalent width of the line, radial velocities of the peaks, intensity of the peaks. To measure the radial velocity we applied Gaussian fitting at the top of the peak. On a few spectra we see three peaks - one violet and two red peaks. In such cases we use the stronger red peak to calculate the distance between the peaks and $\mathrm{V} / \mathrm{R}$ ratio. The measurements are given in a few tables in the Appendix.

\section{RESULTS}

During the period December 2017 - January 2020, the equivalent width of the $H \alpha$ emission line $(W \alpha)$ varies in the range $-26 \AA \leq W \alpha \leq-10 \AA$, the equivalent width of the $H \beta$ emission line $(W \beta)$ varies in the range $-4.4 \AA \leq W \beta \leq-1.4 \AA$, and the equivalent width of the HeI6678 emission line varies in the range $-2.0 \AA \leq W($ HeI6678) $\leq-0.4 \AA$. The variability of the equivalent widths is presented in Fig. 2, together with $\mathrm{V}$ band magnitude and $\mathrm{X}$-ray flux in $2 \mathrm{keV}-10 \mathrm{keV}$. The V-band data are from the American Association of Variable Star Observers (AAVSO). The X-ray data are from MAXI (Matsuoka et al. 2009). The vertical dashed lines indicate the three periods discussed in Sect. 3.2. There is a correlation between equivalent widths of these three lines. The correlation analysis between $W \alpha$ and $W \beta, W \alpha-W($ HeI6678), $W \beta-W($ HeI6678) gives correlation coefficient $\geq 0.80$, significance $p$-value $<10^{-15}$, in other words a very strong correlation between the equivalent widths of the three lines.

It can be seen from Fig. 2 that when the $W_{\alpha}$ decreases the $\mathrm{V}$ band brightness of $\mathrm{X}$ Per increases. The connection between $W_{\alpha}$ and $\mathrm{V}$ band magnitude might be caused by the mass ejection from the Be star as discussed in Sect. 4.1 by $\mathrm{Li}$ et al. (2014). There are no large changes in the X-ray flux. This indicates that the variability of the emission lines during the period December 2017 - January 2020 does not affect the mass accretion rate onto the neutron star.

\subsection{Disc size}

The variability of the distance between the peaks is presented in Fig. 3. The emission lines form in the disc surrounding the Be star. The discs of the Be stars are Keplerian supported 
by the rotation [e.g. Rivinius et al. (2013), Okazaki (2016) and references therein]. For a Keplerian circumstellar disc the peak separation can be regarded as a measure of the outer radius $\left(R_{\text {disc }}\right)$ of the emitting disc (Huang 1972):

$R_{\text {disc }}=R_{1} \frac{(2 v \sin i)^{2}}{\Delta V^{2}}$,

where $R_{1}$ is the radius of the primary and $v \sin i$ is its projected rotational velocity. Radii estimation through this method, is a good approximation for symmetric profiles. The projected rotational velocity of the primary is estimated to be $v \sin i=200 \mathrm{~km} \mathrm{~s}^{-1}$ (Slettebak 1982), $v \sin i=$ $215 \pm 10 \mathrm{~km} \mathrm{~s}^{-1}$ using the HeI $\lambda 4026 \AA$ absorption line (Lyubimkov et al. 1997) and $v \sin i=191 \pm 12 \mathrm{~km} \mathrm{~s}^{-1}$ from the width of the $H \alpha$ emission (Zamanov et al. 2019). For the primary we adopt $R_{1}=10.5 R_{\odot}$ and mass $M_{1}=13.5 M_{\odot}$ (Zamanov et al. 2019). This gives the Keplerian velocity on the surface of the star $V_{K e p l}=495 \mathrm{~km} \mathrm{~s}^{-1}$. Adopting inclination $i=30^{\circ}$ (Delgado-Martí et al. 2001), and that the star rotates with 0.8 of the critical velocity (e.g. Porter \& Rivinius 2003), we estimate $v \sin i \approx 198 \mathrm{~km} \mathrm{~s}^{-1}$, which is in agreement with the above values. This agreement also is a clue that there is no considerable deviation between the orbital plane of the binary and the equatorial plane of the Be star. If it exists at all it should be less then $5^{\circ}$.

On the spectrum 20190325 (see Fig. 1), the HeI emission extends upto $\approx 370 \mathrm{~km} \mathrm{~s}^{-1}$ at zero intensity. We note that velocities in the profiles of the emission lines $>495 \sin i \mathrm{~km} \mathrm{~s}^{-1}$, which is $\approx 250 \mathrm{~km} \mathrm{~s}^{-1}$, are probably super-Keplerian velocities, and indicate eccentric motion in the disc, where these parts of the emission line are formed (see Sect. 3.3).

In December 2017 the emission lines have double-peaked symmetric profiles (see Fig. 1). For 5 spectra obtained in December 2017 we measure $\Delta V \alpha=120 \pm 1 \mathrm{~km} \mathrm{~s}^{-1}, \Delta V \beta=$ $150 \pm 1 \mathrm{~km} \mathrm{~s}^{-1}$, and $\Delta V_{H e I}=180 \pm 2 \mathrm{~km} \mathrm{~s}^{-1}$. Using Eq. 1, these values correspond to disc size $R_{\text {disc }}(H \alpha)=134 R_{\odot}$, $R_{\text {disc }}(H \beta)=86 R_{\odot}$, and $R_{\text {disc }}(H e I 6678)=60 R_{\odot}$. During the period of our observations, the average distance between the peaks of the lines is $\Delta V \alpha=111 \pm 14 \mathrm{~km} \mathrm{~s}^{-1}$, $\Delta V \beta=142 \pm 13 \mathrm{~km} \mathrm{~s}^{-1}, \Delta V_{H e I}=213 \pm 52 \mathrm{~km} \mathrm{~s}^{-1}$. These values correspond to the following average disc size for different emission lines (calculated using Eq. 1): $R_{\text {disc }}(H \alpha)=156 R_{\odot}$, $R_{\text {disc }}(H \beta)=96 R_{\odot}, R_{\text {disc }}\left(\right.$ HeI6678) $=43 R_{\odot}$. The typical errors are about $\pm 5 \%$. For the calculations of the outflowing velocity (Sect. 4.1) we will use $R_{d i s c}(H \beta)=86-96 R_{\odot}$. The average ratios between disc sizes (which is equivalent to the ratio of the peak separations) are: $R_{\text {disc }}(H \alpha) / R_{\text {disc }}(H \beta)=$ $1.62, R_{\text {disc }}(H \alpha) / R_{\text {disc }}(H e I 6678)=3.63$.

\section{$3.2 \mathrm{~V} / \mathrm{R}$ ratio and asymmetries in the disc}

In Fig. 1 it is seen that the emission lines are symmetric in December 2017 , with $V / R$ ratio $\approx 1$. After that all three lines become asymmetric. Fig. 4 presents the $\mathrm{V} / \mathrm{R}$ ratio, calculated as $V / R=\left(I_{V}-1\right) /\left(I_{R}-1\right)$, where $I_{V}$ and $I_{R}$ are the intensity of the blue (violet) and red peak, respectively. The spectra are normalized before the measurements and the continuum level is $\equiv 1.0$.

(1) During the first part of the period (MJD 58095 MJD 58365) in all three lines the V/R-ratio varies in practically the same manner - it decreased from $V / R \approx 1$ to $V / R \approx 0.5$, with speed $\frac{\Delta(V / R)}{\Delta t} \approx 2 \times 10^{-3} \mathrm{~d}^{-1}$. The fact that

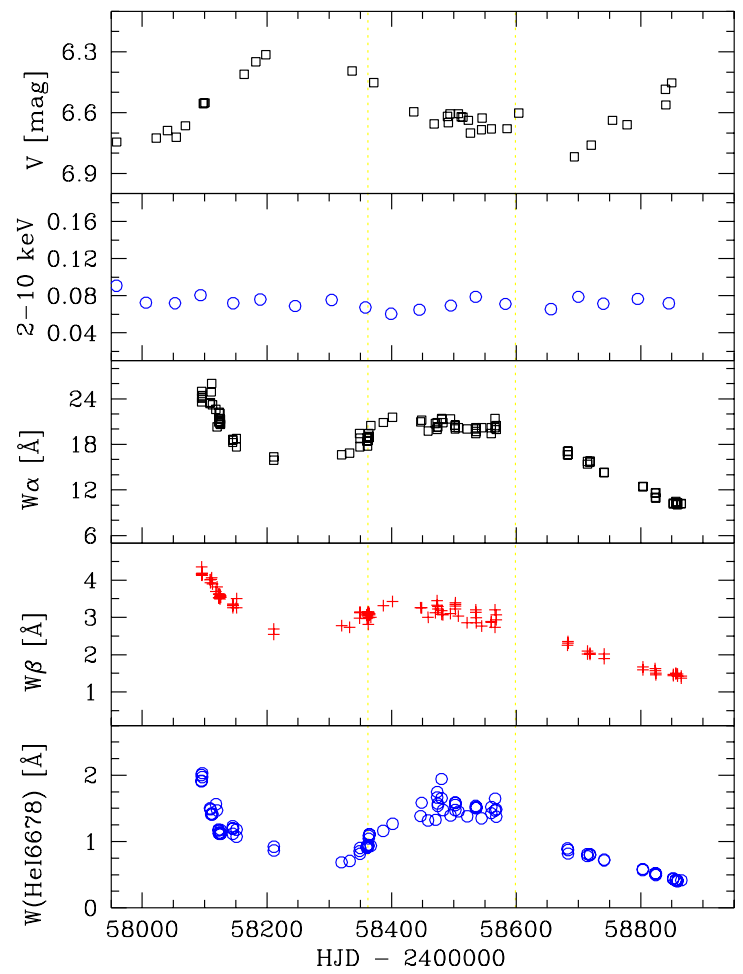

Figure 2. Variability of X Per in V band (AAVSO), 2-10 keV Xrays (MAXI), and the equivalent widths of the emission lines $H \alpha$ (black squares), $H \beta$ (red pluses), and HeI6678 (blue circles).

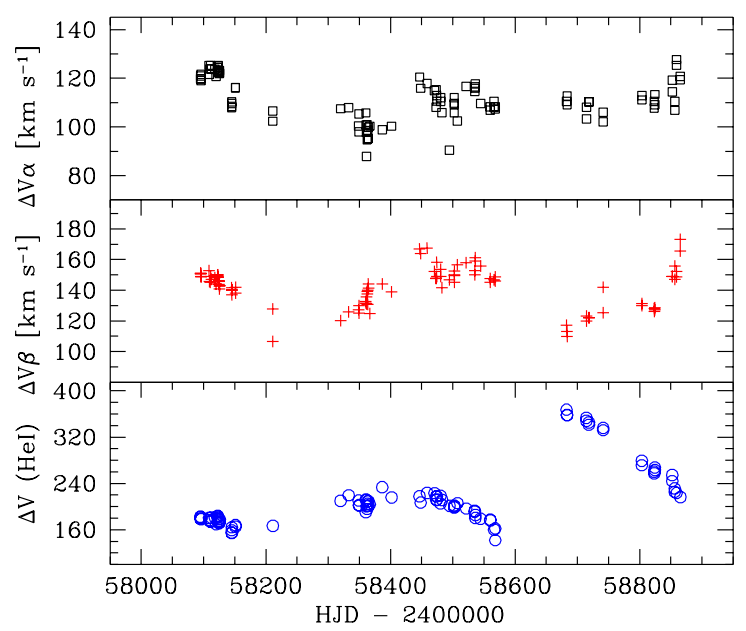

Figure 3. The distance between the peaks of the emission lines $H \alpha$ (black squares), $H \beta$ (red pluses), and HeI6678 (blue circles).

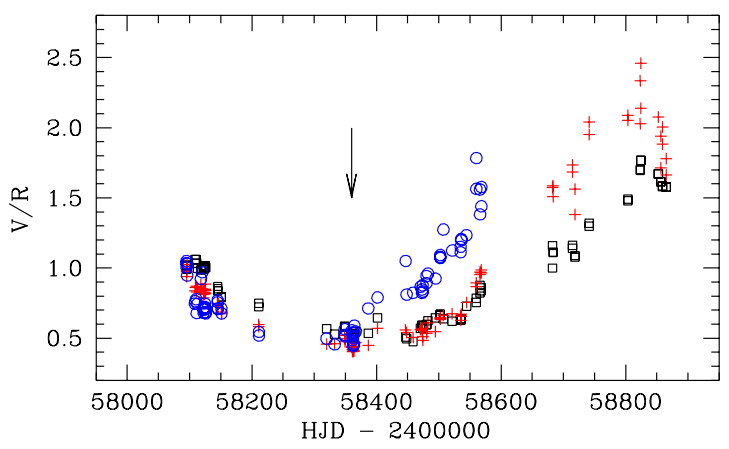

Figure 4. V/R ratio for $H \alpha$ (black squares), $H \beta$ (red pluses), and HeI6678 (blue circles). The arrow indicates the appearance of the eccentric wave. 


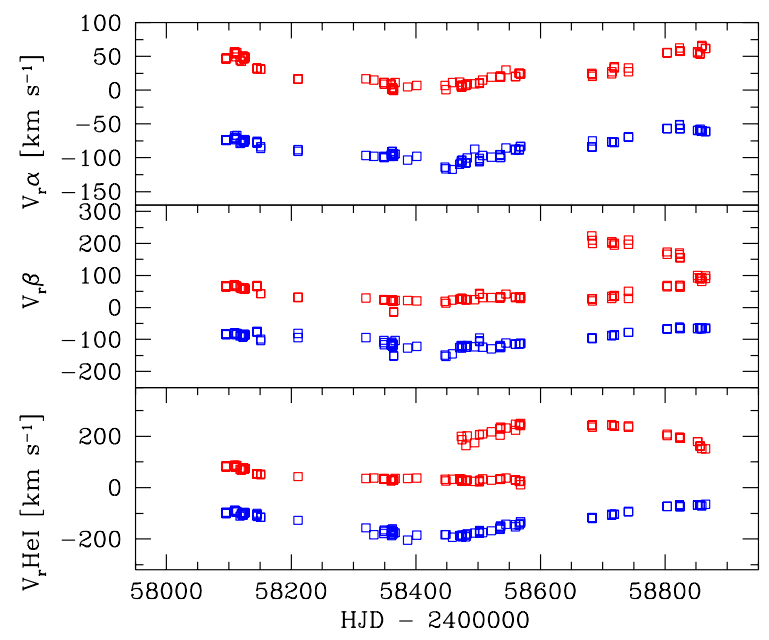

Figure 5. Radial velocities of the peaks.

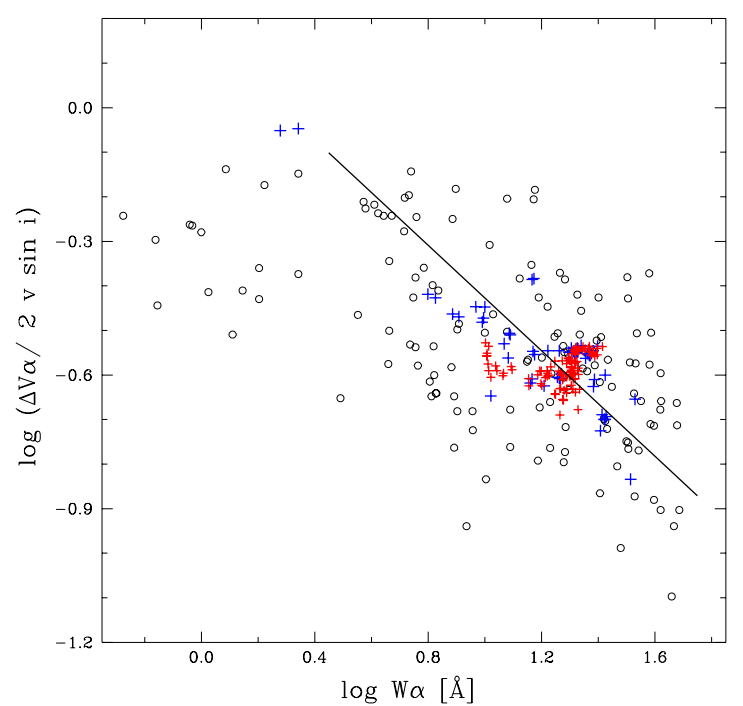

Figure 6. $\Delta V \alpha$ versus $W_{\alpha}$. The circles are the Be stars (see text) and the red crosses are our observations of X Per.
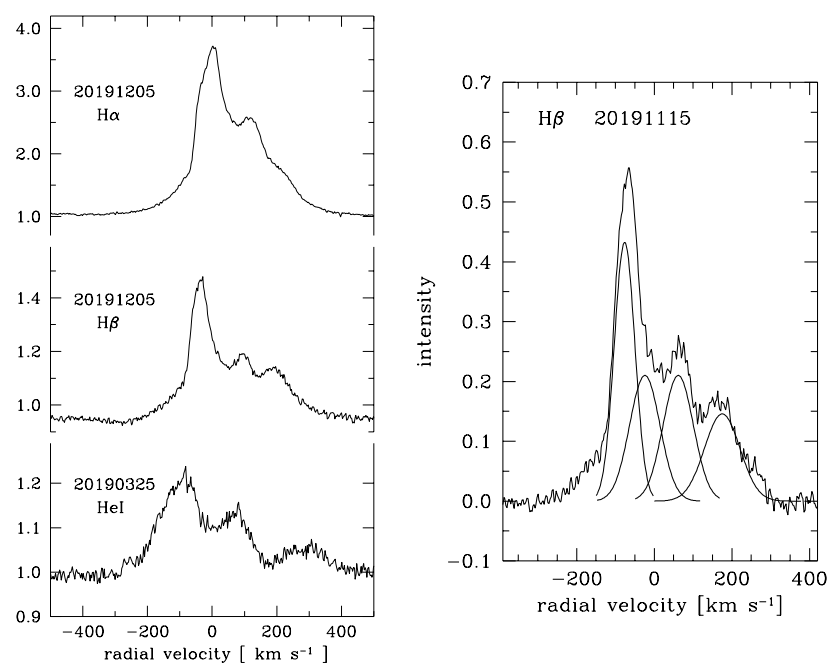

Figure 7. The left panel shows the three component structure of HeI 6678 and $H \beta$ lines, and red shoulder in the $H \alpha$ line. Right panel is an approximation with four Gaussian (see Sect.4.2). the changes in $\mathrm{V} / \mathrm{R}$-ratio are practically identical in all three lines ( $H \alpha, H \beta$ and HeI, see Fig.4) means that the asymmetry develops in the entire disc simultaneously.

(2) During the second part of the period (MJD58365 MJD58600) the V/R-ratio of $H \alpha$ and $H \beta$ goes up from 0.5 to $\sim 1.0$. However the behaviour of the V/R-ratio of HeI6678 deviates from the behaviour of the $\mathrm{H} \alpha$ and $H \beta$ lines (see Fig. 4). V/R (HeI) changes from 0.5 to 1.5 , having a faster rate of change $\frac{\Delta(V / R)}{\Delta t} \approx 4 \times 10^{-3} \mathrm{~d}^{-1}$. For comparison, the $\mathrm{H} \alpha$ emission peaks have rate of change $\frac{\Delta(V / R)}{\Delta t} \approx 1.7 \times 10^{-3}$ $\mathrm{d}^{-1}$ and the $H \beta$ peaks $-\frac{\Delta(V / R)}{\Delta t} \approx 2.3 \times 10^{-3} \mathrm{~d}^{-1}$. In addition to that, a third component appeared in the red side of the HeI profile (Fig. 7). This third component emerged in HeI in November 2018. This is an indication that the structural changes begin in the innermost region of the disc.

(3) The behaviour of the $\mathrm{V} / \mathrm{R}$-ratio of $H \beta$ is similar to that of $H \alpha$ till MJD 58600. After it it begins to deviate (see Fig. 4). This is an indication that the asymmetry spreads out in the disc.

The development of asymmetries visible in the $H \alpha$ peaks corresponds to processes in the outer parts, while the variability of HeI is connected with changes in the inner parts of the circumstellar disc.

\subsection{Third component in He I}

In November 2018 a third component appears in the red side of the HeI line. In March 2019, this component peaks at radial velocity $\sim 250 \mathrm{~km} \mathrm{~s}^{-1}$, and at zero intensity it is in the range $130-390 \mathrm{~km} \mathrm{~s}^{-1}$. The three component structure is demonstrated on Fig. 7, left panel. The Keplerian velocity around the primary is $V_{K e p l}=\sqrt{G M_{1} / r}$, where $G$ is the gravitational constant and $r$ is the distance. We estimate that the observed velocity of the third component corresponds to a distance from the centrum of the primary of $40-20 R_{\odot}$, respectively. Bearing in mind that $R_{1} \approx 10.5 R_{\odot}$, it means that this component originates somewhere about 1-3 stellar radii above the stellar surface. The velocity of this component is similar to the velocities observed during the double disc formation in 1994 (Tarasov \& Roche 1995), when the blue and red peaks of the inner disc were at $-308 \mathrm{~km} \mathrm{~s}^{-1}$ and $+258 \mathrm{~km} \mathrm{~s}^{-1}$, respectively.

\section{ECCENTRIC WAVE}

The discs around stars, or other central massive bodies, can support disturbances in which the fluid motion is nearly Keplerian with non-zero eccentricity. The eccentric waves are expected not only in Be discs but also in accretion discs, protoplanetary discs, and in discs with an embedded planet (e.g. Lynch \& Ogilvie (2019) and references therein). In X Per, the appearance of a significant density enhancement in the disc near the stellar surface and its evolution was also observed and discussed by Tarasov \& Roche (1995) and Clark et al. (2001). Assuming that the observed behaviour of the emission lines of X Per is due to an eccentric wave, we calculate its velocity and eccentricity. 


\subsection{Velocity of the eccentric wave}

We first see the appearance of the third component in HeI lines on our spectrum in November 2018 (20181124). It is not visible on our spectrum obtained in mid-October 2018 (20181010). In March 2019 this component starts to be visible in the red side of $H \beta$. The profile of $H \beta$ on 5 December 2019 (20191205) with three peaks is similar to that of HeI obtained 8 months earlier (20190325), see Fig.7. We suppose that the eccentric wave began somewhere about MJD 58360 (about end of August 2018) when the behaviour of the HeI peaks started to deviate from $\mathrm{H} \beta$ and $\mathrm{H} \alpha$ (see the arrow on Fig. 4). At this time it is likely to be at a distance $r \geq R_{1}$. It reached near to the outer edge of the $H \beta$ disc in January 2020 (when the two red peaks of $H \beta$ blended). This indicates that the eccentric wave spreads from inside out. Bearing in mind the disc size in the lines (Sect. 3.1) this corresponds to an outflowing velocity of the wave $v_{\text {wave }} \approx 1.2 \pm 0.1 \mathrm{~km} \mathrm{~s}^{-1}$.

In the above calculation we supposed that the motion of the density wave is linear. We also supposed that the peak merging corresponds to the moment when the wave reached the outer edge. We performed numerical experiments with Gaussian fitting of the peaks and their blending (merging). The experiments showed that to observe two peaks the difference between their velocities must be $\gtrsim 30 \mathrm{~km} \mathrm{~s}^{-1}$. If it is less than $30 \mathrm{~km} \mathrm{~s}^{-1}$ then we will see one peak. This value depends on the signal-to-noise ratio of the spectra, width of the peaks, their intensity, etc. This limit of $30 \mathrm{~km} \mathrm{~s}^{-1}$ was found with numerical experiment with two Gaussian peaks, having width and intensity similar to those observed. This also indicates that the above estimation should be considered as an upper limit of $v_{\text {wave }}$. Assuming that the merging of the peaks corresponds to the moment when the wave is at $0.85 \pm 0.15$ of the disc size, this will give $v_{\text {wave }} \approx 1.1 \pm 0.2 \mathrm{~km} \mathrm{~s}^{-1}$.

In Fig. 5 are plotted the heliocentric radial velocities of the peaks for $H \alpha\left(V_{r} \alpha\right)$, for $H \beta\left(V_{r} \beta\right)$, and for HeI $6678\left(V_{r} H e I\right)$. The radial velocities of the HeI peaks indicate that the wave begins somewhere about MJD 58350 (August 2018). The radial velocities of the $H \beta$ peaks indicate that the two red peaks merged somewhere about MJD 58850 (January 2020).

In Fig. 6 we plot distance between the peaks of $H \alpha$ normalized with the stellar rotation versus $W \alpha$. These two parameters correlate in the Be stars, representing the fact that the disc grows as $W_{\alpha}$ increases. The black open circles are data for Be stars taken from Andrillat (1983), Hanuschik (1986), Hanuschik et al. (1988), Dachs et al. (1992), Slettebak et al. (1992), and Catanzaro (2013). The red plus signs are our measurements of X Per. We see that X Per is close to the average behaviour of the Be stars. This indicates that the eccentric wave does not change the overall structure of the circumstellar disc.

\subsection{Eccentricity of the eccentric wave}

The vis-viva equation, connects the instantaneous orbital speed of a body at any given point in its trajectory with distance. For periastron and apastron we have:

$v_{p e r}=\sqrt{G M_{1}\left(\frac{2}{a(1-e)}-\frac{1}{a}\right)}$
Table 1. In the table are given date of observations (in the format YYYYMMDD), heliocentric radial velocities of the three peaks, and the calculated eccentricity of the eccentric wave.

\begin{tabular}{lcccc}
\hline & & & & \\
date-obs & $V_{r 1}$ & $V_{r 2}$ & $V_{r 3}$ & $e_{\text {wave }}$ \\
HeI 6678 & & & & \\
20190221 & -147.2 & 29.9 & 231.9 & 0.17 \\
20190323 & -137.7 & 24.8 & 247.8 & 0.23 \\
20190325 & -133.4 & 23.9 & 246.3 & 0.24 \\
20190220 & -155.1 & 29.8 & 249.0 & 0.18 \\
& & & & \\
$H \beta$ & & & & \\
20190819 & -89.6 & 33.0 & 210.2 & 0.33 \\
20190822 & -86.6 & 39.2 & 201.5 & 0.32 \\
20191115 & -67.0 & 62.0 & 176.2 & 0.36 \\
20191206 & -65.8 & 63.6 & 157.6 & 0.31 \\
& & & & \\
H $\alpha$ & & & & \\
20191115 & -57.1 & 59.8 & 145.2 & 0.33 \\
20191115 & -56.9 & 55.0 & 148.6 & 0.36 \\
20191206 & -63.5 & 52.3 & 155.6 & 0.32 \\
& & & & \\
\hline
\end{tabular}

$v_{a p}=\sqrt{G M_{1}\left(\frac{2}{a(1+e)}-\frac{1}{a}\right)}$

where $a$ is the length of the semi-major axis of the elliptical orbit, and $a(1-e)$ and $a(1+e)$ are the distances at which the speed is to be calculated (in our case periastron and apastron, respectively).

Using three spectra on which $H \alpha$ is symmetric (20161211, 20170317, 20171207) we measure the velocity at the half maximum of the $H \alpha$ emission $-11 \pm 3 \mathrm{~km} \mathrm{~s}^{-1}$. The measurement is done using the position of the bisector at the half maximum intensity of the emission, as shown in Fig. 1 of Glebocki et al. (1986). The old spectroscopic data of Hutchings (1977) give an average value for the radial velocity of the Balmer absorption $\approx-20 \mathrm{~km} \mathrm{~s}^{-1}$ and for HeI lines $-19 \pm 10 \mathrm{~km} \mathrm{~s}^{-1}$. Grundstrom et al. (2007) give systemic velocity $\gamma=1.0 \pm 0.9 \mathrm{~km} \mathrm{~s}^{-1}$ on the basis of absorption lines in the IUE spectra. On the spectra where 3 peaks are visible we measure the heliocentric radial velocities of the peaks. They are given in Table 1 . For HeI and $\mathrm{H} \beta$ these are measurements of the peaks. For $H \alpha$ it is the velocity of the shoulder. Correcting these values for the systemic velocity:

$V_{r 1}-\gamma=v_{a p} \sin i$

and

$V_{r 3}-\gamma=v_{\text {per }} \sin i$,

and using Eq. 2 and Eq. 3, we estimate the eccentricity of the wave, which is given in the last column of Table 1. The average eccentricity of the wave is $e_{\text {wave }}=0.29 \pm 0.07$.

In the above calculations we adopted $\gamma=-11 \mathrm{~km} \mathrm{~s}^{-1}$. If we assume $\gamma=-1 \mathrm{~km} \mathrm{~s}^{-1}$, the estimated $e_{\text {wave }}$ will increase up to $e_{\text {wave }}=0.36 \pm 0.07$. There should be two blue peaks corresponding to the two red peaks. In our spectra we see only one, see Fig. 7 (left panel). It means that the two blue peaks are blended (and have approximately equal velocities). To estimate the possible influence of the blending on our value of $e_{\text {wave }}$, we fitted the profile with four Gaussian (Fig. 7, right 
panel). During the fitting we explicitly assumed that the two inner peaks are identical. The fitting suggests that the peak (corresponding to the wave) could be blue shifted with $10-$ $20 \mathrm{~km} \mathrm{~s}^{-1}$. A correction of $-10 \mathrm{~km} \mathrm{~s}^{-1}$ to the measured radial velocity produces a lower value $e_{\text {wave }}=0.24 \pm 0.06$. These two sources of uncertainty give the range $0.16<e_{\text {wave }}<0.42$ for the eccentricity of the wave.

\section{DISCUSSION}

Eccentric discs, in which planet, or star, or fluid elements follow elliptical orbits of variable eccentricity around a central mass, have applications in various astrophysical objects: Be stars (Ogilvie 2008), galactic nuclei (Cao et al. 2018), planetary rings and protoplanetary discs (Lee et al. 2019; Martin et al. 2020). A more deep understanding of the appearance and the evolution of asymmetries and eccentricities in the circumstellar disc of X Per and other Be/X-ray binaries would therefore be of general interest. There are different theories about the origin of the long-term variability observed in Be stars [e.g. Hanuschik et al. (1995), Section 4.2]. The most accepted is the global oscillation scenario, which proposes that a Keplerian disc around a Be stars is subject to global distortion - a one-armed density wave (Okazaki 1991; Savonije \& Heemskerk 1993; Papaloizou \& Savonije 2006), which is an updated and sophisticated version of the old elliptical disc model (Struve 1931). Eccentric mode in Be/Xray binaries can be excited in the disc through direct driving as a result of a one-armed bar potential of the binary (Okazaki et al. 2002).

In our opinion, during the first part or our observations (MJD 58095 - MJD 58365) we observe a global distortion ( $H \alpha, H \beta, H e I$ vary together) similar to that observed in H 1145 - 619 (see Fig. 7 by Alfonso-Garzón et al. (2017)). During the period MJD 58365 - MJD 58865, we observe an eccentric wave, which starts in the innermost parts of the disc and spreads out with velocity $v_{\text {wave }} \approx 1.1 \mathrm{~km} \mathrm{~s}^{-1}$. Once the wave is in the disc, it should begin to rotate. Its rotation is expected to be much slower than the disc rotation (Okazaki \& Kato 1986). The radial velocities on Fig. 5 indicate that the period of rotation of the density wave is probably $\sim 800 \mathrm{~d}$.

The interesting behavior of the HeI emission of X Per was studied by Kunjaya \& Hirata (1995) and by Tarasov \& Roche (1995). They observed four peaks in HeI emission in 1995 and suggested the formation of a double circumstellar disc. Hirata \& Kogure (1977) were first to argue about the existence of a two component structure of the circumstellar envelope around a Be stars following the example of the well known star Pleione (BU Tau). They proposed a model consisting of two layers and also considered a separate fast rotating layer closer to the stellar equator. The formation of a new envelope, coexisting with the previous one is also detected by Nemravová et al. (2010) on the basis of four peak structure of $H \alpha$ emission. Clark et al. (2001) presented an extensive data set of X Per, covering the period 1987 to 2001, and interpreted the HeI variability as a formation of a density wave which moves towards the outer parts faster than the disc formation. It is likely that the four peaks profiles of X Per in 1995 represented a wave with eccentricity almost zero.
Following Delgado-Martí et al. (2001) the periastron passages of the neutron star is expected at

MJD $51353( \pm 7)+250.3( \pm 0.6) \times E$.

The excitation of the eccentric wave could be connected with the periastron passage at about MJD 58361. The periastron passage of the neutron star initiates (generates) a higher tidal wave on the stellar surface. Superposition of a high tide with another mechanism (e.g. non-radial pulsations) could cause a mass ejection event from the star surface into the inner disc. A similar mass ejection was already discussed by Li et al. (2014) in connection between the long term variability of the $H \alpha$ line and the X-ray emission from the neutron star. The recent radiative transfer calculations on the structure of $\mathrm{Be}$ discs in coplanar circular binary systems suggest a V/R cycle every half orbital period (Panoglou et al. 2018). We do not see signs of such modulation in X Per in our data, which covers 770 days, i.e. more than three orbital periods.

\section{CONCLUSIONS}

We report 100 spectral observations of the Be/X-ray binary X Per during the period December 2017 - January 2020. We study the evolution of the profiles of the emission lines $H \alpha$, $H \beta$, and HeI6678, which are formed in the Be circumstellar disc. Their evolution suggests an eccentric wave in the circumstellar disc, for which we find that: (1) it spreads from inside out; (2) its velocity is $v_{\text {wave }}=1.1 \pm 0.2 \mathrm{~km} \mathrm{~s}^{-1}$; (3) the eccentricity of the eccentric wave is in the range $0.17 \leq e_{\text {wave }} \leq 0.41$.

The development of asymmetries in the inner and outer parts of the circumstellar disc in the Be/X-ray binary X Per provides a laboratory to test the theoretical models of eccentric waves in the Be discs. We encourage high-resolution spectroscopic observations of this relatively bright object (e.g. Echelle spectrographs on $2.0 \mathrm{~m}$ class telescopes) to monitor the evolution of the emission lines.

\section{ACKNOWLEDGMENTS}

This work was supported by the Bulgarian National Science Fund project number KП-06-H28/2 08.12.2018 "Binary stars with compact object". The TIGRE telescope is a collaboration of the Hamburger Sternwarte, the Universities of Hamburg, Guanajuato and Liége. UW acknowledges funding by DLR, project 50OR1701. DM acknowledges partial support by grant RD-08-122/2020 from Shumen University. This research has made use of the MAXI data provided by RIKEN, JAXA, and the MAXI team and observations from the AAVSO International Database contributed by observers worldwide. We are very grateful to the anonymous referee for the very helpful and constructive comments on the original manuscript.

Data availability: The spectra are available upon request from the authors: rkz@astro.bas.bg, kstoyanov@astro.bas.bg.

\section{REFERENCES}

Acuner Z., İnam S. Ç., Şahiner Ş., Serim M. M., Baykal A., Swank J., 2014, MNRAS, 444, 457

Alfonso-Garzón J., et al., 2017, A\&A, 607, A52 
Andrillat Y., 1983, A\&AS, 53, 319

Bonev T., et al., 2017, Bulgarian Astronomical Journal, 26, 67

Braes L. L. E., Miley G. K., 1972, Nature, 235, 273

Cao R., Liu F. K., Zhou Z. Q., Komossa S., Ho L. C., 2018, MNRAS, 480, 2929

Catanzaro G., 2013, A\&A, 550, A79

Clark J. S., Tarasov A. E., Okazaki A. T., Roche P., Lyuty V. M., 2001, A\&A, 380, 615

Cowley A. P., McLaughlin D. B., Toney J., MacConnell D. J., 1972, PASP, 84, 834

Dachs J., Hummel W., Hanuschik R. W., 1992, A\&AS, 95, 437

Delgado-Martí H., Levine A. M., Pfahl E., Rappaport S. A., 2001, ApJ, 546, 455

Fabregat J., et al., 1992, A\&A, 259, 522

Glebocki R., Sikorski J., Bielicz E., Krogulec M., 1986, A\&A, 158, 392

Grundstrom E. D., et al., 2007, ApJ, 660, 1398

Hanuschik R. W., 1986, A\&A, 166, 185

Hanuschik R. W., Kozok J. R., Kaiser D., 1988, A\&A, 189, 147

Hanuschik R. W., Hummel W., Dietle O., Sutorius E., 1995, A\&A, 300, 163

Hirata R., Kogure T., 1977, PASJ, 29, 477

Huang S.-S., 1972, ApJ, 171, 549

Kunjaya C., Hirata R., 1995, PASJ, 47, 589

Lee W.-K., Dempsey A. M., Lithwick Y., 2019, ApJ, 882, L11

Li H., Yan J., Zhou J., Liu Q., 2014, AJ, 148, 113

Lynch E. M., Ogilvie G. I., 2019, MNRAS, 488, 1127

Lyubimkov L. S., Rostopchin S. I., Roche P., Tarasov A. E., 1997, MNRAS, 286, 549

Martin R. G., Lissauer J. J., Quarles B., 2020, arXiv e-prints, p. arXiv:2006.05529

Matsuoka M., et al., 2009, PASJ, 61, 999

Nakajima M., Negoro H., Mihara T., Sugizaki M., Yatabe F., Makishima K., 2019, in Oskinova L. M., Bozzo E., Bulik T., Gies D. R., eds, IAU Symposium Vol. 346, IAU Symposium. pp 131-134

Negueruela I., 2007, in St. -Louis N., Moffat A. F. J., eds, Astronomical Society of the Pacific Conference Series Vol. 367, Massive Stars in Interactive Binaries. p. 477

Nemravová J., et al., 2010, A\&A, 516, A80

Ogilvie G. I., 2008, MNRAS, 388, 1372

Okazaki A. T., 1991, PASJ, 43, 75

Okazaki A. T., 2016, in Sigut T. A. A., Jones C. E., eds, Astronomical Society of the Pacific Conference Series Vol. 506, Bright Emissaries: Be Stars as Messengers of Star-Disk Physics. p. 3

Okazaki A. T., Kato S., 1986, Ap\&SS, 119, 109

Okazaki A. T., Bate M. R., Ogilvie G. I., Pringle J. E., 2002, MNRAS, 337, 967

Panoglou D., Faes D. M., Carciofi A. C., Okazaki A. T., Baade D., Rivinius T., Borges Fernandes M., 2018, MNRAS, 473, 3039

Papaloizou J. C. B., Savonije G. J., 2006, A\&A, 456, 1097

Porter J. M., Rivinius T., 2003, PASP, 115, 1153

Reig P., 2011, Ap\&SS, 332, 1

Rivinius T., Carciofi A. C., Martayan C., 2013, A\&ARv, 21, 69

Roche P., et al., 1997, A\&A, 322, 139

Savonije G. J., Heemskerk M. H. M., 1993, A\&A, 276, 409

Schmitt J. H. M. M., et al., 2014, Astronomische Nachrichten, 335, 787

Slettebak A., 1982, ApJS, 50, 55

Slettebak A., Collins II G. W., Truax R., 1992, ApJS, 81, 335

Struve O., 1931, ApJ, 73, 94

Tarasov A. E., Roche P., 1995, MNRAS, 276, L19

Telting J. H., Waters L. B. F. M., Roche P., Boogert A. C. A., Clark J. S., de Martino D., Persi P., 1998, MNRAS, 296, 785

Yatabe F., Makishima K., Mihara T., Nakajima M., Sugizaki M., Kitamoto S., Yoshida Y., Takagi T., 2018, PASJ, 70, 89

Zamanov R., Stoyanov K. A., Wolter U., Marchev D., Petrov N. I., 2019, A\&A, 622, A173

\section{APPENDIX}

This paper has been typeset from a $\mathrm{T}_{\mathrm{E}} \mathrm{X} / \mathrm{LAT}_{\mathrm{E}} \mathrm{X}$ file prepared by the author. 
Table 2. Journal of observations. In the table are given date of observation, observatory, exposure time, Julian day (24400000+).
Table 2. Journal of observations (continuation).

\begin{tabular}{|c|c|c|c|}
\hline 2018-12-21T18:18 & Rozhen & 120 & 58474.26792 \\
\hline 2018-12-21T18:21 & Rozhen & 900 & 58474.26989 \\
\hline 2018-12-27T22:31 & Rozhen & 600 & 58480.44336 \\
\hline 2018-12-27T22:42 & Rozhen & 120 & 58480.45117 \\
\hline 2018-12-30Т07:12 & "LaLuz" & 900 & 58482.80473 \\
\hline 2019-01-11T07:14 & "LaLuz" & 900 & 58494.80544 \\
\hline 2019-01-18T20:16 & Rozhen & 60 & 58502.34774 \\
\hline 2019-01-18T20:19 & Rozhen & 120 & 58502.34988 \\
\hline 2019-01-18T20:23 & Rozhen & 720 & 58502.35273 \\
\hline 2019-01-18T20:37 & Rozhen & 900 & 58502.36252 \\
\hline 2019-01-24Т01:12 & "LaLuz" & 900 & 58507.55335 \\
\hline 2019-02-07Т01:37 & "LaLuz" & 900 & 58521.56939 \\
\hline 2019-02-20T16:41 & Rozhen & 900 & 58535.19549 \\
\hline 2019-02-20T16:57 & Rozhen & 120 & 58535.20681 \\
\hline 2019-02-21T17:38 & Rozhen & 1200 & 58536.23481 \\
\hline 2019-02-21T17:58 & Rozhen & 120 & 58536.24923 \\
\hline 2019-03-02Т01:51 & "LaLuz" & 1125 & 58544.57689 \\
\hline 2019-03-17T18:50 & Rozhen & 600 & 58560.28307 \\
\hline 2019-03-17T19:02 & Rozhen & 120 & 58560.29132 \\
\hline 2019-03-23T19:10 & Rozhen & 120 & 58566.29626 \\
\hline 2019-03-23T19:14 & Rozhen & 900 & 58566.29891 \\
\hline 2019-03-25T18:19 & Rozhen & 120 & 58568.26056 \\
\hline 2019-03-25T18:22 & Rozhen & 900 & 58568.26288 \\
\hline 2019-07-18T00:30 & Rozhen & 600 & 58682.51763 \\
\hline 2019-07-18T00:41 & Rozhen & 120 & 58682.52516 \\
\hline 2019-07-19Т00:51 & Rozhen & 600 & 58683.53222 \\
\hline 2019-07-19Т01:01 & Rozhen & 120 & 58683.53957 \\
\hline 2019-08-19T00:43 & Rozhen & 600 & 58714.52967 \\
\hline 2019-08-19Т00:54 & Rozhen & 1200 & 58714.53737 \\
\hline 2019-08-22T23:06 & Rozhen & 600 & 58718.46281 \\
\hline 2019-08-22T23:18 & Rozhen & 120 & 58718.47082 \\
\hline 2019-09-15Т00:36 & Rozhen & 900 & 58741.52743 \\
\hline 2019-09-15Т00:53 & Rozhen & 120 & 58741.53915 \\
\hline 2019-11-15T22:43 & Rozhen & 900 & 58803.45246 \\
\hline 2019-11-15T22:43 & Rozhen & 900 & 58803.45246 \\
\hline 2019-12-05T20:30 & Rozhen & 600 & 58823.35991 \\
\hline 2019-12-05T20:41 & Rozhen & 120 & 58823.36759 \\
\hline 2019-12-06T19:02 & Rozhen & 600 & 58824.29886 \\
\hline 2019-12-06T19:13 & Rozhen & 120 & 58824.30628 \\
\hline 2020-01-03T17:55 & Rozhen & 600 & 58852.25124 \\
\hline 2020-01-03T18:06 & Rozhen & 120 & 58852.25892 \\
\hline 2020-01-07T16:51 & Rozhen & 1200 & 58856.20631 \\
\hline 2020-01-07T17:12 & Rozhen & 3600 & 58856.22133 \\
\hline 2020-01-10T17:17 & Rozhen & 120 & 58859.22428 \\
\hline 2020-01-10T17:23 & Rozhen & 900 & 58859.22862 \\
\hline 2020-01-16T19:51 & Rozhen & 900 & 58865.33101 \\
\hline 2020-01-16T20:08 & Rozhen & 120 & 58865.34259 \\
\hline
\end{tabular}

\begin{tabular}{|c|c|c|c|}
\hline date-obs & Obs. & $\begin{array}{r}\text { exp-time } \\
\text { [sec] }\end{array}$ & $\mathrm{JD}$ \\
\hline 2015-12-23Т23:16 & Rozhen & 1800 & 57380.47503 \\
\hline 2015-12-23Т23:48 & Rozhen & 600 & 57380.49672 \\
\hline 2015-12-24T20:51 & Rozhen & 600 & 57381.37398 \\
\hline 2015-12-26Т20:01 & Rozhen & 600 & 57383.33892 \\
\hline 2015-12-27Т20:56 & Rozhen & 300 & 57384.37750 \\
\hline 2016-01-30T19:10 & Rozhen & 300 & 57418.30099 \\
\hline 2016-09-23Т01:42 & Rozhen & 600 & 57654.57407 \\
\hline 2016-09-23Т01:42 & Rozhen & 600 & 57654.57407 \\
\hline 2016-12-11T20:58 & Rozhen & 1200 & 57734.37953 \\
\hline 2017-03-17T18:58 & Rozhen & 1200 & 57830.28854 \\
\hline 2017-12-07Т16:57 & Rozhen & 120 & 58095.21181 \\
\hline 2017-12-07T17:03 & Rozhen & 900 & 58095.21620 \\
\hline 2017-12-07T17:20 & Rozhen & 900 & 58095.22811 \\
\hline 2017-12-08T16:45 & Rozhen & 120 & 58096.20389 \\
\hline 2017-12-08Т16:50 & Rozhen & 600 & 58096.20690 \\
\hline 2017-12-21Т03:34 & "LaLuz" & 240 & 58108.65399 \\
\hline 2017-12-22Т00:53 & "LaLuz" & 240 & 58109.54230 \\
\hline 2017-12-23Т00:54 & "LaLuz" & 127 & 58110.54298 \\
\hline 2017-12-24T01:22 & "LaLuz" & 240 & 58111.56198 \\
\hline 2017-12-25Т01:26 & "LaLuz" & 242 & 58112.56500 \\
\hline 2017-12-30Т19:20 & Rozhen & 1200 & 58118.31067 \\
\hline 2018-01-01T17:23 & Rozhen & 1200 & 58120.22886 \\
\hline 2018-01-04T01:28 & "LaLuz" & 900 & 58122.56566 \\
\hline 2018-01-04Т03:56 & "LaLuz" & 900 & 58122.66813 \\
\hline 2018-01-04T06:17 & "LaLuz" & 900 & 58122.76651 \\
\hline 2018-01-05Т01:21 & "LaLuz" & 900 & 58123.56049 \\
\hline 2018-01-06Т01:59 & "LaLuz" & 900 & 58124.58737 \\
\hline 2018-01-06Т04:19 & "LaLuz" & 900 & 58124.68461 \\
\hline 2018-01-06Т06:39 & "LaLuz" & 300 & 58124.78160 \\
\hline 2018-01-07Т01:23 & "LaLuz" & 900 & 58125.56225 \\
\hline 2018-01-07Т03:49 & "LaLuz" & 900 & 58125.66353 \\
\hline 2018-01-07Т06:14 & "LaLuz" & 900 & 58125.76423 \\
\hline 2018-01-26Т17:09 & Rozhen & 120 & 58145.21745 \\
\hline 2018-01-26T17:12 & Rozhen & 180 & 58145.21951 \\
\hline 2018-01-26Т17:16 & Rozhen & 900 & 58145.22261 \\
\hline 2018-01-26T17:32 & Rozhen & 1200 & 58145.23371 \\
\hline 2018-02-01T17:09 & Rozhen & 1200 & 58151.21687 \\
\hline 2018-02-01T17:30 & Rozhen & 300 & 58151.23149 \\
\hline 2018-04-02Т18:42 & Rozhen & 600 & 58211.27615 \\
\hline 2018-04-02T18:53 & Rozhen & 120 & 58211.28358 \\
\hline 2018-07-20T11:09 & "LaLuz" & 600 & 58319.96179 \\
\hline 2018-08-02T11:20 & "LaLuz" & 600 & 58332.97006 \\
\hline 2018-08-18T10:06 & "LaLuz" & 600 & 58348.92028 \\
\hline 2018-08-19Т01:10 & Rozhen & 120 & 58349.54794 \\
\hline 2018-08-19Т01:14 & Rozhen & 900 & 58349.55114 \\
\hline 2018-08-30T00:11 & Rozhen & 600 & 58360.50863 \\
\hline 2018-08-30Т00:23 & Rozhen & 120 & 58360.51631 \\
\hline 2018-08-31Т01:50 & Rozhen & 600 & 58361.57747 \\
\hline 2018-08-31T02:01 & Rozhen & 120 & 58361.58508 \\
\hline 2018-09-01Т01:13 & Rozhen & 600 & 58362.55181 \\
\hline 2018-09-01Т01:25 & Rozhen & 120 & 58362.55977 \\
\hline 2018-09-02Т02:39 & Rozhen & 600 & 58363.61129 \\
\hline 2018-09-02Т02:50 & Rozhen & 120 & 58363.61871 \\
\hline 2018-09-03Т01:21 & Rozhen & 600 & 58364.55719 \\
\hline 2018-09-03Т01:31 & Rozhen & 120 & 58364.56449 \\
\hline 2018-09-05Т08:54 & "LaLuz" & 600 & 58366.87215 \\
\hline 2018-09-25Т08:49 & "LaLuz" & 600 & 58386.87070 \\
\hline 2018-10-10Т07:16 & "LaLuz" & 600 & 58401.80672 \\
\hline 2018-11-24Т06:04 & "LaLuz" & 600 & 58446.75842 \\
\hline 2018-11-25T22:30 & Rozhen & 180 & 58448.44317 \\
\hline 2018-12-06Т07:11 & "LaLuz" & 900 & 58458.80544 \\
\hline 2018-12-18Т07:07 & "LaLuz" & 900 & 58470.80209 \\
\hline 2018-12-20T20:54 & Rozhen & 120 & 58473.37615 \\
\hline QR1/8510000T20:520 & 0円ozhen & 900 & 58473.37847 \\
\hline
\end{tabular}


Table 3. Parameters of the $H \alpha$ emission line. In the table are given Julian day $(2400000+)$, equivalent width, intensity of the violet and red peaks, distance between the peaks, radial velocities of the peaks.

\begin{tabular}{|c|c|c|c|c|c|c|}
\hline JD & $W \alpha$ & $I_{B}$ & $I_{R}$ & $\begin{array}{r}\Delta V \\
{\left[\mathrm{~km} \mathrm{~s}^{-1}\right]}\end{array}$ & $\begin{array}{r}\mathrm{V} \\
{\left[\mathrm{km} \mathrm{s}^{-1}\right]}\end{array}$ & {$\left[\mathrm{km} \mathrm{s}^{-1}\right]$} \\
\hline 58095.21084 & -24.96 & 5.530 & 5.547 & 119.8 & -72.3 & 48.2 \\
\hline 58095.21523 & -24.09 & 5.316 & 5.252 & 121.0 & -72.9 & 48.0 \\
\hline 58095.22714 & -23.61 & 5.365 & 5.316 & 119.7 & -72.7 & 47.7 \\
\hline 58096.20296 & -24.43 & 5.581 & 5.598 & 119.0 & -72.7 & 47.6 \\
\hline 58096.20597 & -24.22 & 5.521 & 5.422 & 121.6 & -73.3 & 49.3 \\
\hline 58118.31064 & -22.61 & 4.954 & 5.006 & 123.7 & -77.5 & 45.9 \\
\hline 58120.22892 & -20.32 & 4.839 & 4.875 & 120.8 & -76.0 & 44.0 \\
\hline 58145.21851 & -18.61 & 4.162 & 4.709 & 108.0 & -74.5 & 33.5 \\
\hline 58145.22057 & -18.67 & 4.146 & 4.755 & 108.4 & -77.0 & 33.9 \\
\hline 58145.22368 & -18.25 & 4.141 & 4.741 & 110.3 & -76.5 & 33.7 \\
\hline 58145.23478 & -18.55 & 4.146 & 4.619 & 109.6 & -76.2 & 33.1 \\
\hline 58151.21816 & -18.77 & 4.076 & 4.859 & 116.2 & -85.1 & 32.5 \\
\hline 58151.23277 & -17.68 & 4.068 & 4.881 & 116.1 & -82.5 & 32.3 \\
\hline 58211.27855 & -16.35 & 3.519 & 4.480 & 106.6 & -89.0 & 17.6 \\
\hline 58211.28598 & -15.92 & 3.628 & 4.511 & 102.4 & -86.8 & 17.9 \\
\hline 58349.54619 & -18.81 & 3.440 & 5.197 & 105.3 & -98.0 & 12.4 \\
\hline 58349.54938 & -17.66 & 3.409 & 5.159 & 98.0 & -98.7 & 10.3 \\
\hline 58360.50660 & -18.42 & 3.278 & 5.383 & 100.7 & -93.8 & 9.2 \\
\hline 58360.51428 & -18.84 & 3.309 & 5.345 & 105.8 & -96.8 & 10.8 \\
\hline 58361.57541 & -17.77 & 3.139 & 5.173 & 97.8 & -90.0 & 3.2 \\
\hline 58361.58303 & -18.42 & 3.158 & 5.258 & 87.9 & -89.5 & 2.9 \\
\hline 58362.54974 & -18.92 & 3.247 & 5.363 & 94.8 & -89.0 & 5.3 \\
\hline 58362.55770 & -18.49 & 3.245 & 5.343 & 100.8 & -92.8 & 3.9 \\
\hline 58363.60919 & -19.45 & 3.292 & 5.438 & 98.5 & -94.8 & 1.5 \\
\hline 58363.61661 & -18.86 & 3.284 & 5.417 & 95.2 & -93.8 & 2.5 \\
\hline 58364.55507 & -18.88 & 3.304 & 5.386 & 95.2 & -96.9 & 2.9 \\
\hline 58364.56238 & -19.03 & 3.293 & 5.419 & 99.9 & -93.5 & 0.8 \\
\hline 58448.44176 & -21.17 & 3.371 & 5.767 & 115.9 & -115.0 & 2.1 \\
\hline 58473.37570 & -19.99 & 3.663 & 5.509 & 108.2 & -103.3 & 7.7 \\
\hline 58473.37802 & -20.87 & 3.607 & 5.397 & 115.2 & -106.1 & 6.3 \\
\hline 58474.26750 & -20.30 & 3.606 & 5.448 & 110.6 & -101.9 & 9.2 \\
\hline 58474.26947 & -20.24 & 3.507 & 5.400 & 112.9 & -105.6 & 5.8 \\
\hline 58480.44321 & -21.40 & 3.710 & 5.538 & 112.0 & -106.1 & 8.1 \\
\hline 58480.45101 & -21.28 & 3.802 & 5.691 & 110.4 & -106.5 & 8.8 \\
\hline 58502.34850 & -20.51 & 3.822 & 5.251 & 105.8 & -99.7 & 11.6 \\
\hline 58502.35064 & -20.05 & 3.836 & 5.252 & 109.7 & -104.7 & 12.1 \\
\hline 58502.35348 & -20.54 & 3.809 & 5.196 & 109.3 & -103.1 & 12.0 \\
\hline 58502.36328 & -20.25 & 3.833 & 5.222 & 112.0 & -102.5 & 11.7 \\
\hline 58535.19736 & -19.42 & 3.714 & 5.307 & 114.6 & -94.8 & 21.8 \\
\hline 58535.20868 & -20.20 & 3.747 & 5.353 & 115.9 & -94.4 & 20.4 \\
\hline 58536.23671 & -20.01 & 3.678 & 5.219 & 116.5 & -97.8 & 21.7 \\
\hline 58536.25113 & -19.68 & 3.743 & 5.286 & 117.6 & -98.7 & 20.8 \\
\hline 58560.28540 & -20.20 & 3.905 & 4.708 & 108.3 & -87.3 & 21.9 \\
\hline 58560.29365 & -19.43 & 3.893 & 4.837 & 106.9 & -85.9 & 21.0 \\
\hline 58566.29864 & -21.40 & 4.367 & 4.845 & 108.3 & -87.6 & 26.7 \\
\hline 58566.30129 & -20.18 & 4.117 & 4.788 & 110.6 & -86.3 & 25.8 \\
\hline 58568.26295 & -19.94 & 4.175 & 4.693 & 107.3 & -82.3 & 25.3 \\
\hline 58568.26527 & -20.45 & 4.093 & 4.686 & 108.3 & -81.8 & 25.4 \\
\hline 58108.65545 & -23.37 & 5.518 & 5.257 & 125.3 & -69.8 & 56.3 \\
\hline 58109.54377 & -23.51 & 5.421 & 5.273 & 121.6 & -66.1 & 58.4 \\
\hline 58110.54374 & -24.88 & 5.477 & 5.217 & 123.8 & -69.0 & 55.1 \\
\hline 58111.56345 & -26.02 & 5.385 & 5.244 & 125.2 & -72.0 & 50.6 \\
\hline 58112.56647 & -23.20 & 5.046 & 5.056 & 123.9 & -65.5 & 57.3 \\
\hline 58122.57100 & -20.95 & 4.840 & 4.850 & 124.3 & -72.3 & 51.6 \\
\hline 58122.67347 & -21.42 & 4.890 & 4.931 & 123.4 & -73.8 & 50.4 \\
\hline 58122.77185 & -21.03 & 4.859 & 4.884 & 124.6 & -74.0 & 50.4 \\
\hline 58123.56582 & -22.24 & 5.003 & 5.008 & 125.2 & -72.7 & 51.3 \\
\hline
\end{tabular}


Table 3. continuation.

\begin{tabular}{|c|c|c|c|c|c|c|}
\hline 58124.59271 & -22.08 & 4.983 & 4.921 & 122.4 & -74.1 & 49.0 \\
\hline 58124.68994 & -20.87 & 4.933 & 4.892 & 122.4 & -74.8 & 47.8 \\
\hline 58124.78338 & -20.99 & 4.921 & 4.856 & 122.6 & -75.1 & 48.5 \\
\hline 58125.56759 & -20.60 & 4.849 & 4.829 & 123.2 & -72.0 & 50.9 \\
\hline 58125.66887 & -20.71 & 4.888 & 4.872 & 122.0 & -72.9 & 49.2 \\
\hline 58125.76956 & -21.29 & 4.902 & 4.887 & 122.2 & -73.7 & 49.6 \\
\hline 58319.96541 & -16.63 & 3.143 & 4.770 & 107.5 & -95.4 & 18.2 \\
\hline 58332.97367 & -16.86 & 3.144 & 5.053 & 107.9 & -96.7 & 16.2 \\
\hline 58348.92388 & -19.44 & 3.499 & 5.272 & 100.4 & -96.5 & 12.7 \\
\hline 58366.87575 & -20.47 & 3.436 & 5.451 & 100.3 & -93.3 & 12.4 \\
\hline 58386.87432 & -20.88 & 3.508 & 5.680 & 98.9 & -102.3 & 6.1 \\
\hline 58401.81034 & -21.58 & 3.908 & 5.515 & 100.3 & -96.5 & 8.1 \\
\hline 58446.76209 & -20.95 & 3.400 & 5.717 & 120.5 & -112.6 & 8.3 \\
\hline 58458.81081 & -19.76 & 3.172 & 5.571 & 117.9 & -116.1 & 12.3 \\
\hline 58470.80746 & -20.75 & 3.496 & 5.368 & 114.9 & -108.5 & 13.3 \\
\hline 58482.81011 & -20.85 & 3.744 & 5.403 & 105.9 & -99.0 & 10.7 \\
\hline 58494.81083 & -21.35 & 3.815 & 5.372 & 90.4 & -85.8 & 10.6 \\
\hline 58507.55874 & -20.20 & 3.730 & 5.266 & 102.4 & -95.2 & 16.5 \\
\hline 58521.57478 & -20.01 & 3.636 & 5.246 & 116.7 & -98.1 & 20.6 \\
\hline 58544.58365 & -20.18 & 3.939 & 5.038 & 109.6 & -84.3 & 31.4 \\
\hline 58682.52460 & -16.63 & 4.196 & 3.755 & 110.5 & -82.3 & 26.0 \\
\hline 58682.52940 & -17.08 & - & - & - & - & - \\
\hline 58683.53920 & -17.11 & 4.182 & 3.864 & 112.7 & -83.0 & 24.2 \\
\hline 58683.54370 & -16.64 & 4.261 & 3.931 & 109.1 & -73.7 & 21.7 \\
\hline 58714.53390 & -15.77 & 3.998 & 3.579 & 103.3 & -75.1 & 25.5 \\
\hline 58714.54510 & -15.39 & 4.002 & 3.633 & 108.1 & -75.1 & 28.5 \\
\hline 58718.46660 & -15.81 & 3.925 & 3.686 & 110.3 & -75.6 & 34.2 \\
\hline 58718.47190 & -15.64 & 3.951 & 3.740 & 110.3 & -76.4 & 36.0 \\
\hline 58741.53080 & -14.26 & 4.135 & 3.377 & 106.1 & -68.5 & 34.3 \\
\hline 58741.53800 & -14.31 & 4.158 & 3.432 & 102.1 & -67.5 & 28.7 \\
\hline 58803.45210 & -12.39 & 3.829 & 2.898 & 112.9 & -55.4 & 57.2 \\
\hline 58803.45210 & -12.49 & 3.835 & 2.916 & 111.1 & -55.7 & 56.4 \\
\hline 58823.35790 & -11.58 & 3.637 & 2.550 & 107.7 & -50.6 & 59.7 \\
\hline 58823.36280 & -11.03 & 3.625 & 2.548 & 110.5 & -49.6 & 63.9 \\
\hline 58824.29680 & -11.66 & 3.621 & 2.482 & 109.1 & -55.1 & 58.6 \\
\hline 58824.30150 & -10.92 & 3.618 & 2.481 & 113.3 & -55.8 & 59.7 \\
\hline 58852.25040 & -10.15 & 3.294 & 2.374 & 119.2 & -58.2 & 56.4 \\
\hline 58852.25530 & -10.26 & 3.339 & 2.398 & 114.4 & -58.1 & 58.1 \\
\hline 58856.20920 & -10.51 & 3.251 & 2.395 & 106.8 & -56.3 & 54.8 \\
\hline 58856.23810 & -10.32 & 3.179 & 2.347 & 110.5 & -58.6 & 55.1 \\
\hline 58859.22110 & -10.33 & 3.176 & 2.372 & 125.3 & -58.7 & 67.4 \\
\hline 58859.23000 & -10.06 & 3.153 & 2.362 & 127.7 & -58.8 & 65.6 \\
\hline 58865.33290 & -10.20 & 3.161 & 2.370 & 120.8 & -59.7 & 63.1 \\
\hline 58865.33990 & -10.19 & 3.231 & 2.414 & 119.4 & -60.4 & 62.9 \\
\hline
\end{tabular}


Table 4. Parameters of the $H \beta$ emission line. In the table are given Julian day $(2400000+)$, equivalent width, intensity of the violet and red peaks, distance between the peaks.

\begin{tabular}{|c|c|c|c|c|c|c|c|}
\hline 58095.21084 & -4.357 & 2.094 & 2.167 & 150.5 & -87.3 & 61.7 & - \\
\hline 58095.21523 & -4.140 & 2.092 & 2.115 & 150.9 & -86.5 & 64.2 & - \\
\hline 58095.22714 & -4.172 & 2.088 & 2.116 & 151.0 & -87.3 & 63.9 & - \\
\hline 58096.20296 & -4.132 & 2.161 & 2.131 & 148.9 & -85.8 & 63.3 & - \\
\hline 58096.20597 & -4.184 & 2.122 & 2.115 & 148.8 & -88.1 & 61.7 & - \\
\hline 58118.31064 & -3.699 & 1.965 & 2.094 & 148.1 & -91.9 & 58.7 & - \\
\hline 58120.22892 & -3.814 & 1.944 & 2.106 & 143.9 & -87.9 & 56.7 & - \\
\hline 58145.21851 & -3.262 & 1.775 & 2.039 & 141.5 & -78.7 & 65.7 & - \\
\hline 58145.22057 & -3.315 & 1.763 & 2.048 & 137.0 & -78.1 & 62.8 & - \\
\hline 58145.22368 & -3.322 & 1.754 & 2.015 & 140.1 & -78.9 & 63.3 & - \\
\hline 58145.23478 & -3.360 & 1.762 & 2.023 & 140.3 & -80.2 & 62.5 & - \\
\hline 58151.21816 & -3.259 & 1.725 & 2.074 & 138.0 & -102.0 & 40.0 & - \\
\hline 58151.23277 & -3.509 & 1.747 & 2.088 & 141.8 & -107.1 & 40.0 & - \\
\hline 58211.27855 & -2.544 & 1.548 & 1.918 & 106.5 & -84.4 & 28.8 & - \\
\hline 58211.28598 & -2.683 & 1.545 & 1.936 & 127.6 & -97.2 & 28.0 & - \\
\hline 58349.54619 & -3.123 & 1.520 & 2.095 & 125.0 & -119.1 & 19.3 & - \\
\hline 58349.54938 & -3.150 & 1.505 & 2.018 & 130.0 & -113.1 & 21.7 & - \\
\hline 58360.50660 & -2.963 & 1.449 & 2.035 & 132.7 & -120.0 & 19.6 & - \\
\hline 58360.51428 & -3.062 & 1.469 & 2.059 & 131.5 & -115.7 & 18.7 & - \\
\hline 58361.57541 & -3.034 & 1.413 & 2.021 & 131.2 & -116.9 & 19.6 & - \\
\hline 58361.58303 & -3.096 & 1.437 & 2.040 & 135.7 & -111.8 & 20.4 & - \\
\hline 58362.54974 & -2.815 & 1.439 & 2.072 & 137.4 & -116.8 & 17.9 & - \\
\hline 58362.55770 & -3.151 & 1.447 & 2.062 & 139.1 & -124.8 & 19.3 & - \\
\hline 58363.60919 & -3.133 & 1.434 & 2.066 & 140.0 & -121.7 & 18.4 & - \\
\hline 58363.61661 & -2.993 & 1.437 & 2.068 & 130.8 & -117.9 & 16.0 & - \\
\hline 58364.55507 & -3.103 & 1.460 & 2.038 & 141.3 & -155.3 & -18.6 & - \\
\hline 58364.56238 & -3.072 & 1.475 & 2.023 & 144.1 & -153.9 & -16.1 & - \\
\hline 58448.44176 & -3.263 & 1.552 & 2.021 & 163.8 & -155.9 & 10.1 & - \\
\hline 58473.37570 & -3.328 & 1.576 & 2.012 & 147.7 & -122.6 & 25.2 & - \\
\hline 58473.37802 & -3.450 & 1.548 & 1.985 & 147.8 & -123.4 & 22.2 & - \\
\hline 58474.26750 & -3.288 & 1.535 & 2.106 & 158.2 & -130.1 & 25.0 & - \\
\hline 58474.26947 & -3.219 & 1.515 & 2.010 & 148.9 & -124.8 & 23.5 & - \\
\hline 58480.44321 & -3.179 & 1.549 & 2.005 & 153.6 & -127.9 & 21.5 & - \\
\hline 58480.45101 & -3.078 & 1.549 & 2.026 & 148.9 & -126.9 & 20.6 & - \\
\hline 58502.34850 & -3.392 & 1.597 & 1.933 & 145.1 & -99.0 & 40.1 & - \\
\hline 58502.35064 & -3.229 & 1.590 & 1.910 & 150.2 & -107.6 & 39.4 & - \\
\hline 58502.35348 & -3.347 & 1.601 & 1.932 & 149.4 & -108.9 & 41.1 & - \\
\hline 58502.36328 & -3.307 & 1.588 & 1.923 & 152.4 & -109.7 & 40.1 & - \\
\hline 58535.19736 & -3.198 & 1.569 & 1.866 & 150.0 & -122.2 & 27.5 & - \\
\hline 58535.20868 & -3.131 & 1.590 & 1.941 & 152.8 & -125.8 & 26.1 & - \\
\hline 58536.23671 & -2.858 & 1.574 & 1.875 & 158.9 & -128.8 & 29.1 & - \\
\hline 58536.25113 & -2.988 & 1.601 & 1.897 & 161.1 & -127.4 & 29.4 & - \\
\hline 58560.28540 & -2.870 & 1.629 & 1.726 & 147.6 & -118.7 & 29.5 & - \\
\hline 58560.29365 & -2.885 & 1.661 & 1.739 & 145.0 & -118.6 & 27.7 & - \\
\hline 58566.29864 & -3.198 & 1.772 & 1.809 & 146.9 & -117.2 & 28.4 & - \\
\hline 58566.30129 & -2.738 & 1.672 & 1.691 & - & - & - & - \\
\hline 58568.26295 & -2.934 & 1.706 & 1.732 & 148.7 & -115.8 & 25.1 & - \\
\hline 58568.26527 & -3.073 & 1.698 & 1.707 & 146.0 & -115.5 & 30.4 & - \\
\hline 58108.65545 & -4.003 & 1.969 & 2.157 & 152.7 & -84.4 & 68.1 & - \\
\hline 58109.54377 & -3.924 & 1.971 & 2.126 & 145.7 & -83.1 & 62.5 & - \\
\hline 58110.54374 & -4.017 & 2.109 & 2.273 & 145.2 & - & - & - \\
\hline 58111.56345 & -4.062 & 1.999 & 2.206 & 149.0 & -88.7 & 63.9 & - \\
\hline 58112.56647 & -3.892 & 2.006 & 2.168 & 147.0 & -84.4 & 63.1 & - \\
\hline 58122.57100 & -3.621 & 1.835 & 2.059 & 148.4 & -94.9 & 58.0 & - \\
\hline 58122.67347 & -3.521 & 1.892 & 2.053 & 149.8 & -91.5 & 56.0 & - \\
\hline 58122.77185 & -3.525 & 1.845 & 2.039 & 150.0 & -94.5 & 57.7 & - \\
\hline 58123.56582 & -3.613 & 1.880 & 2.062 & 148.7 & -90.5 & 57.6 & - \\
\hline 58124.59271 & -3.615 & 1.899 & 2.066 & 146.3 & -87.6 & 58.1 & - \\
\hline 58124.68994 & -3.488 & 1.868 & 2.023 & 145.8 & -89.9 & 54.9 & - \\
\hline 58124.78338 & -3.548 & 1.855 & 2.047 & 143.8 & -86.6 & 55.0 & - \\
\hline
\end{tabular}


Table 4. continuation.

\begin{tabular}{|c|c|c|c|c|c|c|c|}
\hline 58125.56759 & -3.582 & 1.871 & 2.059 & 140.7 & -89.7 & 53.3 & - \\
\hline 58125.66887 & -3.569 & 1.917 & 2.035 & 142.8 & -89.2 & 54.6 & - \\
\hline 58125.76956 & -3.541 & 1.859 & 2.021 & 143.0 & -88.7 & 55.5 & - \\
\hline 58319.96541 & -2.779 & 1.442 & 1.966 & 120.2 & -97.5 & 26.2 & - \\
\hline 58332.97367 & -2.733 & 1.450 & 1.980 & 126.0 & - & - & - \\
\hline 58348.92388 & -2.979 & 1.536 & 2.031 & 127.2 & -106.0 & 21.4 & - \\
\hline 58366.87575 & -3.002 & 1.505 & 2.076 & 124.7 & -106.5 & 18.9 & - \\
\hline 58386.87432 & -3.309 & 1.474 & 2.056 & 144.2 & -130.4 & 18.4 & - \\
\hline 58401.81034 & -3.424 & 1.598 & 2.047 & 139.0 & -124.3 & 17.0 & - \\
\hline 58446.76209 & -3.257 & 1.578 & 2.034 & 166.9 & -152.5 & 16.2 & - \\
\hline 58458.81081 & -3.001 & 1.509 & 2.006 & 167.5 & -147.9 & 20.1 & - \\
\hline 58470.80746 & -3.126 & 1.541 & 1.964 & 152.3 & -128.5 & 23.2 & - \\
\hline 58482.81011 & -3.067 & 1.556 & 1.911 & 141.6 & -121.9 & 21.7 & - \\
\hline 58494.81083 & -3.104 & 1.523 & 1.956 & 146.7 & -126.4 & 21.1 & - \\
\hline 58507.55874 & -3.032 & 1.565 & 1.852 & 156.7 & -127.8 & 27.3 & - \\
\hline 58521.57478 & -2.855 & 1.567 & 1.842 & 157.9 & -132.4 & 27.8 & - \\
\hline 58544.58365 & -2.764 & 1.584 & 1.773 & 155.7 & -114.4 & 38.8 & - \\
\hline 58682.52460 & -2.246 & 1.630 & 1.400 & 117.1 & -98.3 & 23.3 & 220.0 \\
\hline 58682.52940 & - & - & - & - & - & - & - \\
\hline 58683.53920 & -2.309 & 1.598 & 1.377 & 113.2 & -100.5 & 23.8 & 206.6 \\
\hline 58683.54370 & -2.355 & 1.604 & 1.400 & 109.8 & -100.4 & 17.1 & 196.9 \\
\hline 58714.53390 & -2.100 & 1.619 & 1.357 & 123.3 & -90.8 & 24.9 & 202.2 \\
\hline 58714.54510 & -2.015 & 1.603 & 1.358 & 119.9 & -91.5 & 31.5 & 197.5 \\
\hline 58718.46660 & -2.039 & 1.608 & 1.389 & 122.0 & -89.8 & 34.2 & 197.7 \\
\hline 58718.47190 & -2.009 & 1.598 & 1.433 & 122.2 & -89.7 & 31.0 & 191.5 \\
\hline 58741.53080 & -1.896 & 1.615 & 1.315 & 125.3 & -81.0 & 47.2 & 207.0 \\
\hline 58741.53800 & -2.019 & 1.661 & 1.324 & 141.8 & -81.2 & 24.8 & 194.1 \\
\hline 58803.45210 & -1.675 & 1.536 & 1.261 & 131.4 & -70.8 & 65.4 & 170.1 \\
\hline 58803.46410 & -1.594 & 1.518 & 1.248 & 130.0 & -70.2 & 61.8 & 163.1 \\
\hline 58823.35790 & -1.571 & 1.502 & 1.215 & 128.1 & -65.7 & 65.9 & 162.5 \\
\hline 58823.36280 & -1.628 & 1.509 & 1.251 & 126.2 & -65.4 & 63.7 & 166.7 \\
\hline 58824.29680 & -1.456 & 1.497 & 1.202 & 128.7 & -68.7 & 65.2 & 153.4 \\
\hline 58824.30150 & -1.507 & 1.479 & 1.224 & 127.3 & -68.8 & 60.1 & 151.3 \\
\hline 58852.25040 & -1.438 & 1.439 & 1.119 & 149.0 & -68.7 & 89.0 & - \\
\hline 58852.25530 & -1.473 & 1.467 & 1.225 & 148.9 & -69.1 & 96.8 & - \\
\hline 58856.20920 & -1.498 & 1.415 & 1.242 & 155.8 & -67.9 & 88.3 & - \\
\hline 58856.23810 & -1.481 & 1.429 & 1.221 & 147.2 & -68.4 & 86.5 & - \\
\hline 58859.22110 & -1.475 & 1.422 & 1.224 & 152.3 & -68.7 & 89.3 & - \\
\hline 58859.23000 & -1.427 & 1.421 & 1.210 & 148.7 & -68.6 & 78.7 & - \\
\hline 58865.33290 & -1.427 & 1.434 & 1.244 & 173.3 & -67.6 & 96.1 & - \\
\hline 58865.33990 & -1.366 & 1.431 & 1.259 & 165.5 & -69.4 & 87.3 & - \\
\hline
\end{tabular}


Table 5. Parameters of the HeI 6678 emission line. In the table are given Julian day (2400000+), equivalent width, intensity of the violet and red peaks, distance between the peaks, radial velocities of the peaks.

\begin{tabular}{|c|c|c|c|c|c|c|c|}
\hline JD & $W \alpha$ & $I_{B}$ & $I_{R}$ & $\begin{array}{r}\Delta V \\
{\left[\mathrm{~km} \mathrm{~s}^{-1}\right]}\end{array}$ & $\begin{array}{r}\mathrm{V} \\
{\left[\mathrm{km} \mathrm{s}^{-1}\right]}\end{array}$ & $\begin{array}{r}\mathrm{V} \\
{\left[\mathrm{km} \mathrm{s}^{-1}\right]}\end{array}$ & $\begin{array}{r}\mathrm{V} \\
{\left[\mathrm{km} \mathrm{s}^{-1}\right]}\end{array}$ \\
\hline 58095.21084 & -2.004 & 1.363 & 1.346 & 181.9 & -98.0 & 81.7 & \\
\hline 58095.21523 & -1.908 & 1.334 & 1.323 & 182.5 & -95.5 & 84.2 & \\
\hline 58095.22714 & -1.917 & 1.327 & 1.325 & 178.9 & -100.2 & 82.7 & \\
\hline 58096.20296 & -2.030 & 1.344 & 1.363 & 178.3 & -101.0 & 81.0 & \\
\hline 58096.20597 & -1.973 & 1.340 & 1.330 & 179.3 & -98.3 & 84.8 & \\
\hline 58118.31064 & -1.564 & 1.278 & 1.301 & 180.9 & -111.3 & 71.2 & \\
\hline 58120.22892 & -1.470 & 1.264 & 1.272 & 169.3 & -102.8 & 67.8 & \\
\hline 58145.21851 & -1.118 & 1.185 & 1.240 & 159.1 & -105.6 & 52.8 & \\
\hline 58145.22057 & -1.229 & 1.179 & 1.251 & 154.6 & -99.8 & 53.6 & \\
\hline 58145.22368 & -1.193 & 1.174 & 1.246 & 155.1 & -106.6 & 53.1 & \\
\hline 58145.23478 & -1.202 & 1.183 & 1.242 & 164.2 & -111.5 & 54.2 & \\
\hline 58151.21816 & -1.180 & 1.178 & 1.263 & 165.3 & -116.1 & 49.5 & \\
\hline 58151.23277 & -1.073 & 1.193 & 1.271 & 168.0 & -115.8 & 52.8 & \\
\hline 58211.27855 & -0.865 & 1.107 & 1.206 & 166.8 & -127.2 & 43.1 & \\
\hline 58211.28598 & -0.922 & 1.114 & 1.209 & - & - & - & \\
\hline 58349.54619 & -0.904 & 1.110 & 1.210 & 201.8 & -164.5 & 34.3 & \\
\hline 58349.54938 & -0.816 & 1.100 & 1.195 & 202.8 & -169.8 & 35.3 & \\
\hline 58360.50660 & -0.932 & 1.109 & 1.196 & 212.0 & -181.8 & 29.9 & \\
\hline 58360.51428 & -0.907 & 1.113 & 1.209 & 190.3 & -165.4 & 26.0 & \\
\hline 58361.57541 & -0.939 & 1.109 & 1.212 & 211.7 & -185.9 & 29.1 & \\
\hline 58361.58303 & -0.911 & 1.106 & 1.218 & 200.5 & -173.1 & 28.8 & \\
\hline 58362.54974 & -0.927 & 1.095 & 1.214 & 201.2 & -167.9 & 30.2 & \\
\hline 58362.55770 & -0.950 & 1.103 & 1.205 & 196.0 & -161.1 & 31.5 & \\
\hline 58363.60919 & -1.044 & 1.099 & 1.219 & 209.0 & -179.9 & 29.8 & \\
\hline 58363.61661 & -1.089 & 1.107 & 1.231 & 204.9 & -172.4 & 29.9 & \\
\hline 58364.55507 & -1.112 & 1.107 & 1.200 & 209.7 & -173.5 & 28.8 & \\
\hline 58364.56238 & -1.104 & 1.121 & 1.205 & 201.8 & -172.6 & 30.6 & \\
\hline 58448.44176 & -1.586 & 1.197 & 1.243 & 206.9 & -182.7 & 25.4 & \\
\hline 58473.37570 & -1.749 & 1.201 & 1.244 & 217.6 & -189.1 & 32.5 & \\
\hline 58473.37802 & -1.665 & 1.178 & 1.210 & 211.5 & -188.1 & 27.2 & 199.1 \\
\hline 58474.26750 & -1.540 & 1.218 & 1.262 & 212.2 & -185.4 & 25.3 & - \\
\hline 58474.26947 & -1.573 & 1.186 & 1.212 & 218.3 & -190.9 & 26.5 & 187.0 \\
\hline 58480.44321 & -1.653 & 1.214 & 1.227 & 218.8 & -190.9 & 28.0 & 163.3 \\
\hline 58480.45101 & -1.942 & 1.265 & 1.297 & 205.2 & -185.5 & 26.1 & - \\
\hline 58502.34850 & -1.550 & 1.233 & 1.216 & 199.9 & -176.6 & 22.1 & - \\
\hline 58502.35064 & -1.580 & 1.208 & 1.194 & 201.7 & -171.3 & 28.6 & - \\
\hline 58502.35348 & -1.475 & 1.212 & 1.197 & 198.4 & -166.7 & 28.0 & 205.8 \\
\hline 58502.36328 & -1.591 & 1.211 & 1.193 & 201.7 & -172.5 & 27.5 & 206.8 \\
\hline 58535.19736 & -1.505 & 1.205 & 1.178 & 191.2 & -157.2 & 32.2 & 231.0 \\
\hline 58535.20868 & -1.536 & 1.217 & 1.195 & 192.6 & -162.1 & 34.6 & 204.6 \\
\hline 58536.23671 & -1.505 & 1.205 & 1.171 & 180.2 & -149.2 & 34.5 & 229.0 \\
\hline 58536.25113 & -1.522 & 1.221 & 1.183 & 185.4 & -152.7 & 31.8 & 234.8 \\
\hline 58560.28540 & -1.427 & 1.216 & 1.138 & 177.4 & -144.8 & 30.0 & 246.5 \\
\hline 58560.29365 & -1.520 & 1.232 & 1.130 & 176.2 & -152.9 & 28.7 & 223.3 \\
\hline 58566.29864 & -1.648 & 1.242 & 1.175 & -1.0 & - & - & - \\
\hline 58566.30129 & -1.470 & 1.220 & 1.141 & 160.2 & -144.8 & 23.5 & 245.2 \\
\hline 58568.26295 & -1.486 & 1.229 & 1.159 & 141.9 & -138.9 & 11.1 & 250.1 \\
\hline 58568.26527 & -1.375 & 1.224 & 1.142 & 162.2 & -133.3 & 25.5 & 240.9 \\
\hline 58108.65545 & -1.501 & 1.233 & 1.312 & 180.5 & -94.5 & 84.2 & \\
\hline 58109.54377 & -1.485 & 1.240 & 1.314 & 177.0 & -87.3 & 87.2 & \\
\hline 58110.54374 & -1.417 & 1.246 & 1.316 & 174.6 & -90.6 & 80.2 & \\
\hline 58111.56345 & -1.406 & 1.214 & 1.315 & 174.1 & -92.9 & 80.1 & \\
\hline 58112.56647 & -1.417 & 1.247 & 1.332 & 181.2 & -94.4 & 85.8 & \\
\hline 58122.57100 & -1.180 & 1.199 & 1.292 & 181.0 & -105.4 & 75.1 & \\
\hline 58122.67347 & -1.165 & 1.197 & 1.280 & 183.2 & -107.5 & 73.5 & \\
\hline 58122.77185 & -1.120 & 1.198 & 1.280 & 184.3 & -105.2 & 75.2 & \\
\hline 58123.56582 & -1.129 & 1.199 & 1.276 & 178.9 & -100.1 & 76.1 & \\
\hline
\end{tabular}


Table 5. continuation.

\begin{tabular}{|c|c|c|c|c|c|c|c|}
\hline 58124.59271 & -1.115 & 1.198 & 1.265 & 173.3 & -98.1 & 74.1 & \\
\hline 58124.68994 & -1.113 & 1.197 & 1.273 & 170.8 & -98.3 & 73.4 & \\
\hline 58124.78338 & -1.134 & 1.200 & 1.281 & 172.2 & -97.6 & 71.7 & \\
\hline 58125.56759 & -1.114 & 1.190 & 1.277 & 173.0 & -97.2 & 74.3 & \\
\hline 58125.66887 & -1.176 & 1.190 & 1.281 & 175.5 & -97.3 & 73.8 & \\
\hline 58125.76956 & -1.124 & 1.191 & 1.268 & 178.0 & -99.8 & 73.1 & \\
\hline 58319.96541 & -0.687 & 1.074 & 1.149 & 209.9 & -156.8 & 35.3 & \\
\hline 58332.97367 & -0.707 & 1.078 & 1.171 & 219.8 & -182.9 & 37.3 & \\
\hline 58348.92388 & -0.857 & 1.105 & 1.186 & 210.5 & -178.2 & 32.3 & \\
\hline 58366.87575 & -0.936 & 1.108 & 1.197 & 203.8 & -176.2 & 35.9 & \\
\hline 58386.87432 & -1.162 & 1.141 & 1.198 & 233.5 & -204.5 & 35.7 & \\
\hline 58401.81034 & -1.269 & 1.166 & 1.210 & 215.9 & -184.4 & 36.9 & \\
\hline 58446.76209 & -1.383 & 1.207 & 1.197 & 217.8 & -183.2 & 32.8 & \\
\hline 58458.81081 & -1.319 & 1.159 & 1.193 & 223.6 & -192.5 & 32.1 & \\
\hline 58470.80746 & -1.326 & 1.170 & 1.196 & 223.3 & -185.8 & 35.0 & \\
\hline 58482.81011 & -1.475 & 1.196 & 1.204 & 211.6 & -179.6 & 30.0 & 201.7 \\
\hline 58494.81083 & -1.394 & 1.187 & 1.202 & 201.5 & -177.1 & 24.7 & 174.4 \\
\hline 58507.55874 & -1.454 & 1.218 & 1.171 & 206.1 & -174.9 & 33.9 & 208.6 \\
\hline 58521.57478 & -1.381 & 1.188 & 1.167 & 196.6 & -167.8 & 28.4 & 217.5 \\
\hline 58544.58365 & -1.351 & 1.206 & 1.167 & 179.0 & -142.5 & 36.9 & 232.3 \\
\hline 58682.52460 & -0.896 & 1.223 & 1.089 & 367.4 & -117.1 & 242.3 & \\
\hline 58683.53920 & -0.873 & 1.229 & 1.081 & 357.9 & -120.7 & 235.8 & \\
\hline 58683.54370 & -0.817 & 1.228 & 1.106 & 358.4 & -116.9 & 244.8 & \\
\hline 58714.53390 & -0.813 & 1.180 & 1.088 & 348.2 & -104.4 & 242.5 & \\
\hline 58714.54510 & -0.781 & 1.176 & 1.083 & 353.4 & -107.6 & 244.7 & \\
\hline 58718.46660 & -0.796 & 1.182 & 1.081 & 345.4 & -104.5 & 239.8 & \\
\hline 58718.47190 & -0.808 & 1.192 & 1.104 & 341.3 & -103.1 & 240.1 & \\
\hline 58741.53080 & -0.716 & 1.197 & 1.081 & 331.9 & -95.1 & 240.6 & \\
\hline 58741.53800 & -0.727 & 1.191 & 1.091 & 335.8 & -92.9 & 235.8 & \\
\hline 58803.45210 & -0.586 & 1.129 & 1.092 & 279.2 & -72.6 & 208.5 & \\
\hline 58803.46410 & -0.570 & 1.120 & 1.091 & 271.5 & -72.6 & 201.9 & \\
\hline 58823.35790 & -0.510 & 1.115 & 1.080 & 257.3 & -67.3 & 195.5 & \\
\hline 58823.36280 & -0.518 & 1.122 & 1.091 & 260.1 & -67.6 & 197.3 & \\
\hline 58824.29680 & -0.521 & 1.118 & 1.092 & 263.3 & -73.2 & 192.4 & \\
\hline 58824.30150 & -0.494 & 1.121 & 1.094 & 267.6 & -74.4 & 192.9 & \\
\hline 58852.25040 & -0.436 & 1.110 & 1.069 & 244.1 & -67.8 & 179.2 & \\
\hline 58852.25530 & -0.446 & 1.118 & 1.079 & 254.5 & -68.4 & 179.4 & \\
\hline 58856.20920 & -0.422 & 1.107 & 1.081 & 225.4 & -67.6 & 160.1 & \\
\hline 58856.23810 & -0.413 & 1.088 & 1.087 & 231.4 & -67.2 & 163.2 & \\
\hline 58859.22110 & -0.401 & - & - & - & - & - & \\
\hline 58859.23000 & -0.417 & 1.098 & 1.073 & 223.4 & -70.8 & 152.7 & \\
\hline 58865.33290 & -0.417 & 1.086 & 1.078 & 216.4 & -64.9 & 150.5 & \\
\hline
\end{tabular}

